\title{
Reducing allograft contamination and disease transmission: intraosseous temperatures of femoral head allografts during autoclaving
}

\author{
Chay-You Ang ${ }^{1}$, mBBs, Mrcs, Andy Khye-Soon $\underline{\text { Yew }}^{1}$, PhD, Darren Keng-Jin $\underline{T a y}^{1}$, MBBS, FRCs, Shi-Lu Chia ${ }^{1}$, FRCs, PhD,
} Seng-Jin $\underline{Y e O}^{1}$, MBBS, FRCS, Ngai-Nung $\underline{\mathrm{LO}}^{1}$, MBBS, FRCS, Pak-Lin $\underline{C h i n^{1}}$, MBBS, FRCSE

\begin{abstract}
INTRODUCTION The Singapore General Hospital Bone Bank, which exclusively stores femoral head allografts, relies on flash sterilisation to prevent allograft-related disease transmission and wound infection. However, intraosseous temperatures during autoclaving may be lower than required to eliminate human immunodeficiency virus, and hepatitis $B$ and $C$ viruses. The aim of this study is to determine the intraosseous temperatures of femoral head allografts during autoclaving and to assess the adequacy of autoclaving in preventing disease transmission.

METHODS Six femoral heads were acquired from patients who underwent hip arthroplasty. The specimens were divided into two groups. The first group underwent flash sterilisation with a sterilisation time of 4 min, while a longer sterilisation time of 22 min was used for the second group.

RESULTS The highest core temperature in the first group was $130^{\circ} \mathrm{C}$, while the core temperatures in the second group plateaued at $133^{\circ} \mathrm{C}$ for all allografts. In the first group, only smaller allografts maintained temperatures sufficient for the inactivation of the clinically relevant viral pathogens. In contrast, all allografts in the second group were terminally sterilised. CONCLUSION There is an inverse correlation between the size of allografts and intraosseous temperatures achieved during autoclaving. Therefore, we recommend dividing large allografts into smaller pieces, in order to achieve intraosseous temperatures adequate for the elimination of transmissible pathogens during flash sterilisation. Allografts should not be terminally sterilised, as the resulting allografts will become unusable. Despite modern processing techniques, stringent donor selection remains vital in the effort to prevent allograft-related infections. Autoclaving is an economical and efficacious method of preventing allograft-related disease transmission.
\end{abstract}

Keywords: allografts, autoclaving, bone bank, disease transmission, femoral heads

\section{INTRODUCTION}

Bone grafts, which can be broadly classified as autografts and allografts, are frequently used during orthopaedic surgeries to reconstruct bony defects and augment healing. However, autografts remain the gold standard since there is complete histocompatibility and no risk of disease transmission with this donor source. The osteogenic, osteoconductive and osteoinductive properties of the donor bone are fully retained when it is utilised as a fresh autograft. Hence, if available, autografts are the preferred choice in reconstructive surgeries. However, the use of autografts is frequently limited by its supply, especially in the paediatric population and in major reconstructive procedures involving large defects. ${ }^{(1)}$ The other major drawback is donor site morbidity, which includes infection, prolonged wound drainage, haematomas, reoperation, chronic pain, sensory loss and unsightly scars. ${ }^{(1,2)}$

Allografts, on the other hand, are plentiful and avoid donor site morbidity. However, allografts carry the risk of disease transmission, with contaminated allografts possibly containing blood-borne pathogens and resulting in wound infections. In order to mitigate these problems, the American Association of Tissue Banks has recommended a set of criteria for selecting donors, in addition to serological testing for certain infectious diseases. ${ }^{(3)}$ Allograft processing, which commonly uses methods such as gamma irradiation, heat treatment, chemical disinfection or a combination of methods, further reduces these risks. ${ }^{(1,4)}$

Since its establishment in 1981, the Singapore General Hospital (SGH) Bone Bank, Singapore, has exclusively stored femoral head allografts and relied on flash sterilisation as the primary method for reducing allograft-related disease transmission and wound infection. Routine serological testing for human immunodeficiency virus (HIV), and hepatitis B and C viruses was only implemented later. ${ }^{(5)}$ The SGH Bone Bank Protocol has since been validated in a previous study, in which the method used by SGH was shown to maintain the sterility of autoclaved allografts for up to six months. ${ }^{(5)}$

Autoclaving is a widely available and economical processing method, which effectively decontaminates allografts through the sterilisation of exterior surfaces. However, Böhm and Stihler have reported that intraosseous temperatures during autoclaving are significantly lower than expected, and thus may not be sufficient to eradicate transmissible pathogens. ${ }^{(6)}$ Nonetheless, as that study was conducted using entire calf femurs, the results may not be applicable to femoral head allografts, which are considerably smaller. To the best of our knowledge, there has not been any published literature regarding the intraosseous temperatures of femoral head allografts during the autoclaving process. Thus, the aim of the present study was to determine the intraosseous temperatures of femoral 
head allografts during autoclaving and to assess the adequacy of autoclaving in the prevention of disease transmission.

\section{METHODS}

In September 2012, we acquired six femoral heads from patients who underwent hip arthroplasty as a result of trauma or osteoarthritis. The diameters of the femoral heads were measured in both the coronal and transverse planes using callipers, and the averages of both values were recorded.

A $2.5-\mathrm{mm}$ drill was used to create a tunnel in each femoral head and a thermocouple (2-mm General Electric Type T thermocouple; General Electric, Fairfield, CT, USA) was subsequently introduced into it. Each tunnel was drilled perpendicularly to a depth that corresponds to half the measured diameter (i.e. the radius) of the femoral head. This ensures that the thermocouple will be placed near the centre of the femoral head, thus providing accurate measurements of its core temperature. To insulate the thermocouple from the external environment, the drill hole was sealed using a heat-resistant polyimide tape (3M Polyimide Film Tape 5413; 3M, St Paul, MN, USA), as shown in Fig. 1. The specimens were then placed in individual containers and autoclaved in a Getinge HS66 series steam steriliser (Getinge HS6617; Getinge, Sweden). Core temperatures were measured and recorded during the autoclaving process using the Kaye Validator 2000 (General Electric, Fairfield, CT, USA).

The six femoral heads were divided into two groups, with each group containing three femoral head allografts. The first group of femoral heads underwent a flash sterilisation procedure similar to that described in the SGH Bone Bank Protocol, ${ }^{(5)}$ but with a slightly longer sterilisation time of $4 \mathrm{~min}$, as per the recommendation in the Centers for Disease Control and Prevention (CDC) guidelines for porous objects. ${ }^{(7)}$ The second group underwent three cycles of pre-vacuum, with a longer sterilisation time of $22 \mathrm{~min}$, in order to determine the minimum time required to completely sterilise the specimens.

This study was approved by the institutional review board of our institution and all specimens were disposed of after completion of the study.

\section{RESULTS}

In the group of femoral heads that underwent flash sterilisation, the highest recorded core temperature of $130^{\circ} \mathrm{C}$ was in a 44.0-mm femoral head. The remaining 43.0-mm and 47.8-mm femoral heads recorded maximum temperatures of $126^{\circ} \mathrm{C}$ and $104^{\circ} \mathrm{C}$, respectively. Fig. 2 shows the temperature graphs for the allografts in this group of femoral heads, which were similar and displayed a progressive upward trend. This suggests that there was no leakage, and accurate measurements of the intraosseous temperatures were obtained.

When the sterilisation time was increased to $22 \mathrm{~min}$, the core temperatures for all the allografts plateaued at $133^{\circ} \mathrm{C}$ (Fig. 3). However, the temperature waveform for the 48.0-mm allograft suggests that there could have been a leak in the seal, as there were aberrant spikes in temperature after the second pre-vacuum. In both groups, none of the femoral heads reached $134^{\circ} \mathrm{C}$.

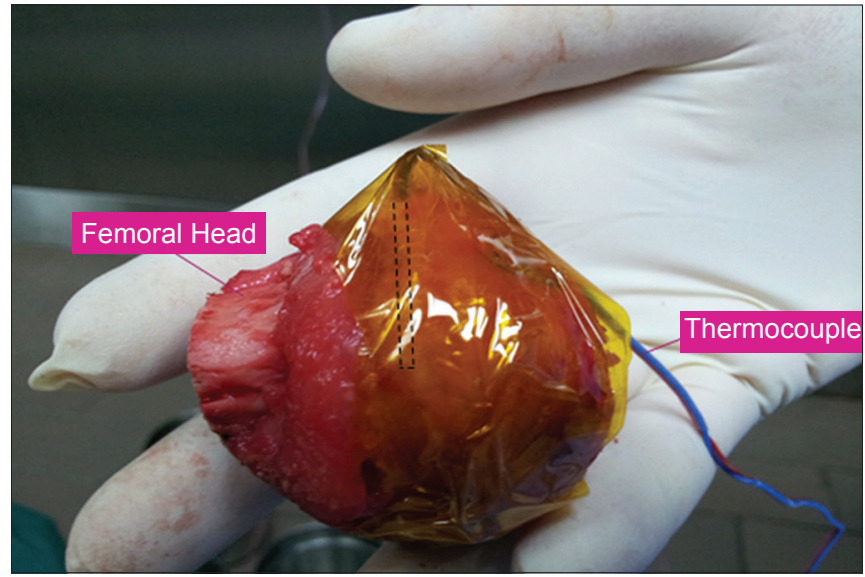

Fig. 1 Photograph shows a 2-mm Type T thermocouple inserted into the core of the femoral head and sealed using heat-resistant polyimide tape. The dotted lines represent the depth of insertion of the thermocouple.

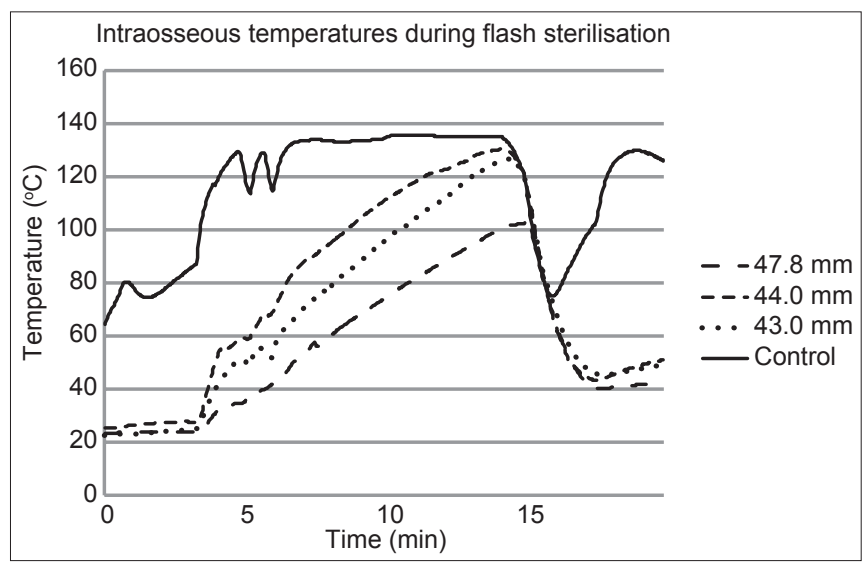

Fig. 2 Temperature graphs show the intraosseous temperatures of the femoral heads during autoclaving, with a sterilisation time of $4 \mathrm{~min}$ and a single pre-vacuum cycle. The graphs of all the femoral heads are similar, suggesting that there was no leakage of steam into the drill hole, and thus, accurate intraosseous temperatures were obtained.

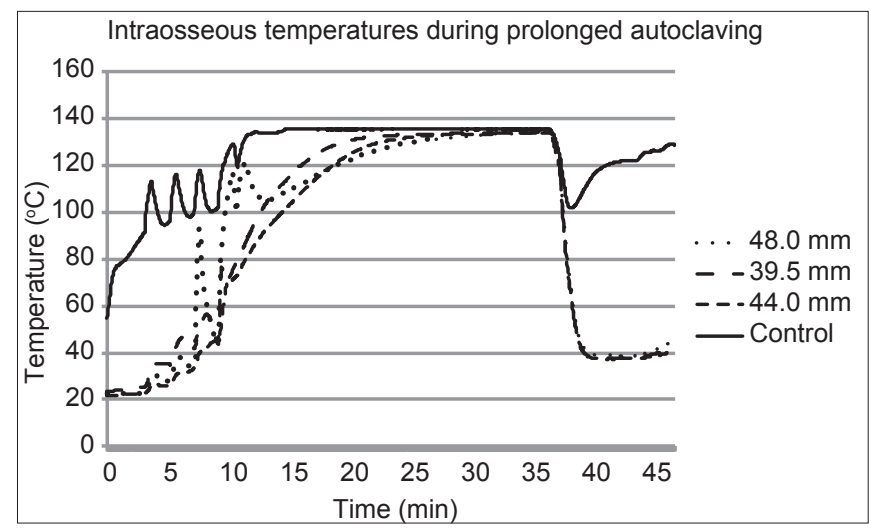

Fig. 3 Temperature graphs show the intraosseous temperatures of the femoral heads during autoclaving with a sterilisation time of $22 \mathrm{~min}$ and three pre-vacuum cycles. The temperature waveform for the $48.0-\mathrm{mm}$ allograft suggests that there may have been a leak in the seal, as there were aberrant spikes in temperature.

\section{DISCUSSION}

The transmission of blood-borne pathogens is a rare but devastating consequence of allograft use. Therefore, many bone banks currently adopt stringent donor selection criteria and routinely perform screening blood tests in order to prevent disease 
transmission. However, despite such measures, there have been reported cases of HIV and hepatitis C transmission as a result of allograft transplantation. ${ }^{(8,9)}$ Although the donors in those cases had initial negative screening test results, they were later tested positive using more sensitive methods. It is important to note that in those cases, the allografts used did not undergo additional processing or treatment. ${ }^{(9)}$ To date, there has been no reported case of disease transmission via the use of processed bone allografts.

Autoclaving is the most reliable and widely used method of sterilising medical equipment. Flash sterilisation refers to the autoclaving of unwrapped objects, which allows the objects to come into direct and rapid contact with steam, leading to a shorter sterilisation time. When the SGH Bone Bank was set up, flash sterilisation was used to reduce the risk of infection, while limiting the thermal damage to allografts. Although flash sterilisation is efficacious in reducing the incidence of allograft contamination, its effectiveness in preventing the transmission of blood-borne pathogens remains unknown. ${ }^{(5)}$ The hepatitis B virus is known to have high heat resistance, requiring a temperature of $98^{\circ} \mathrm{C}$ sustained for a minimum of 2 min to inactivate it. ${ }^{(10)} \mathrm{On}$ the other hand, HIV is heat-sensitive and easily destroyed at the intraosseous temperatures attained in the present study. ${ }^{(11)}$ The hepatitis $\mathrm{C}$ virus has a higher resistance to heat than HIV, but can be inactivated after 4 min at $65^{\circ} \mathrm{C} .{ }^{(12)}$

In the present study, only femoral head allografts with diameters of up to $44.0 \mathrm{~mm}$ were able to maintain temperatures of $98^{\circ} \mathrm{C}$ or higher for at least 2 min during flash sterilisation (Fig. 2). This is despite the use of a slightly longer sterilisation time of 4 min, implying that flash sterilisation alone may not be adequate to prevent the transmission of hepatitis B in larger allografts. However, in the prevention of hepatitis $C$ transmission, our results showed that flash sterilisation is adequate for allografts with diameters measuring up to $47.8 \mathrm{~mm}$. On the other hand, when a sterilisation time of 22 min was used, we were able to achieve sterilisation of the allografts according to the CDC guidelines. ${ }^{(7)}$ However, this resulted in significant damage to the allografts, rendering them unfit for use.

It is reasonable to assume an inverse correlation between the size of the allografts and the intraosseous temperatures achieved during autoclaving. This is because larger allografts have higher masses and therefore require more thermal energy and a longer time to heat up. In the present study, the diameter of the femoral head was used as a surrogate measurement for the mass of the allograft, as callipers are more readily available than weighing machines in operating theatres. However, the diameter of the femoral head may not correlate well with the mass of the allograft. Depending on the amount of remnant femoral neck, allografts with similar head diameters may have significantly different masses. This possibility may account for the higher intraosseous temperature observed in the $44.0-\mathrm{mm}$ specimen than in the 43.0-mm specimen in the flash sterilisation group (Fig. 2).

It should be noted that in the present study, all six allografts were obtained from elderly patients whose bone mineral density were likely to fall within the osteoporotic range. Therefore, the
Table I. Prices of bone grafts and potential substitutes.

\begin{tabular}{lc}
\hline Bone graft/substitute & Price (SGD) \\
\hline Tutoplast cancellous block & 1,659 \\
$(20 \mathrm{~mm} \times 30 \mathrm{~mm} \times 12 \mathrm{~mm})$ & 958 \\
Tutoplast bone chips $15 \mathrm{cc}$ & 326 \\
ChronOS B-TCP granules $10 \mathrm{cc}$ & $150-300^{*}$ \\
SGH femoral head allograft
\end{tabular}

*Fully subsidised patients pay SGD 150 , whereas private patients are charged SGD 300. SGH: Singapore General Hospital

results of the present study may not be applicable to allografts obtained from patients with normal bone density.

Compared to commercial products, autoclaved femoral heads remain the most economical source of bone graft at our institute (Table I). This is especially the case in situations where large amounts of allografts are required. In such cases, the cost savings in using autoclaved femoral heads can be substantial.

In conclusion, stringent donor selection using modern screening tests remains a vital step in the prevention of allograft-related infections. Processing allografts further reduces the risk of infection, and as shown in the present study, flash sterilisation is only suitable for the prevention of disease transmission in smaller allografts. We recommend that large allografts be divided into smaller pieces prior to flash sterilisation so that the intraosseous temperatures achieved are enough to eliminate transmissible pathogens. Although prolonged autoclaving ensures the sterility of allografts, it is likely to result in their destruction. Therefore, flash sterilisation is favoured over terminal sterilisation, as it is capable of preventing disease transmission while preserving the integrity of the allografts.

\section{REFERENCES}

1. Khan SN, Cammisa FP Jr, Sandhu HS, et al. The biology of bone grafting. J Am Acad Orthop Surg 2005; 13:77-86.

2. Younger EM, Chapman MW. Morbidity at bone graft donor sites. J Orthop Trauma 1989; 3:192-5.

3. Zwitser EW, Jiya TU, George Licher H, van Royen BJ. Design and management of an orthopaedic bone bank in The Netherlands. Cell Tissue Bank 2012; 13:63-9.

4. Galia CR, Moreira LF. The Biology of Bone Grafts. In: Fokter SK, ed. Recent Advances in Arthroplasty. InTech, 2012.

5. Ang CY, Loh DS, Chaw HW, Chin PL. Simple novel bone bank storage: the Singapore General Hospital experience. Biopreserv Biobank 2012; 10:526-8.

6. Böhm P, Stihler J. Intraosseous temperature during autoclaving. J Bone Joint Surg Br 1995; 77:649-53.

7. Rutala WA, Weber DJ, Healthcare Infection Control Practices Advisory Committee (HICPAC). Guideline for Disinfection and Sterilization in Healthcare Facilities, 2008. USA: Centers for Disease Control and Prevention, 2008.

8. Simonds RJ, Holmberg SD, Hurwitz RL, et al. Transmission of human immunodeficiency virus type 1 from a seronegative organ and tissue donor. N Engl J Med 1992; 326:726-32.

9. Centers for Disease Control and Prevention (CDC). Hepatitis C virus transmission from an antibody-negative organ and tissue donor--United States, 2000-2002. MMWR Morb Mortal Wkly Rep 2003; 52:273-4, 276.

10. Kobayashi H, Tsuzuki M, Koshimizu K, et al. Susceptibility of hepatitis B virus to disinfectants or heat. J Clin Microbiol 1984; 20:214-6.

11. Charm SE, Landau S, Williams B, et al. High-temperature short-time heat inactivation of HIV and other viruses in human blood plasma. Vox Sang $1992 ; 62: 12-20$

12. Song $\mathrm{H}, \mathrm{Li}$ J, Shi S, et al. Thermal stability and inactivation of hepatitis $\mathrm{C}$ virus grown in cell culture. Virol J 2010; 7:40. 